\title{
A Markov Dilation of a Non-Quasifree Bloch Evolution
}

\section{B. Kümmerer and W. Schröder *}

Mathematisches Institut, Universität Tübingen, Auf der Morgenstelle 10, D-7400 Tübingen, Federal Republic of Germany

\begin{abstract}
We construct a new minimal dilation of a dynamical system governed by a Bloch equation. In contrast to a dilation of the same dynamical system recently obtained by Varilly [13] our dilation satisfies a Markov property. This presents the first example of a Markov dilation for a noncommutative dynamical system which is not equivalent to a quasifree evolution. Furthermore the dilation turns out to be a generalized $K$-system.
\end{abstract}

\section{Introduction}

In the operator algebraic framework of quantum statistical mechanics we define a (continuous) dynamical system by a triple $(\mathfrak{M}, \psi, \tau)$, where $\mathfrak{M}$ is a $W^{*}$-algebra, $\psi$ is a faithful normal state on $\mathfrak{M}$, and $\tau$ is a (pointwise weak* continuous) representation of the semigroup $\mathbb{N}$ (respectively $\mathbb{R}_{+}$) as completely positive identity preserving operators on $\mathfrak{M}$ leaving $\psi$ invariant. In particular, if these operators are *-automorphisms, $(\mathfrak{M}, \psi, \tau)$ will be called a conservative dynamical system.

Given a dynamical system $(\mathfrak{M}, \psi, \tau)$, various reasons raise the problem of constructing a conservative dynamical system $(\hat{\mathfrak{M}}, \hat{\psi}, \hat{\tau})$ containing it in the following sense. There is an injective *-representation $i: \mathfrak{M} \rightarrow \hat{\mathfrak{M}}$ and a projection $E$ of norm one of $\hat{\mathfrak{M}}$ onto $i(\mathfrak{M})$ such that the diagram

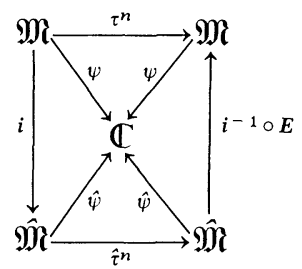

* Research supported in part by Studienstiftung des deutschen Volkes 
commutes for all $n \in \mathbb{N}_{0}$. Then $(\hat{\mathfrak{M}}, \hat{\psi}, \hat{\tau} ; i, E)$ is said to be a dilation of $(\mathfrak{M}, \psi, \tau)$. The dilation is called minimal if $\bigvee_{k \in \mathbb{Z}} \hat{\tau}^{k}(i(\mathfrak{M}))=\hat{\mathfrak{M}}$; here and henceforth for any family $\left(\mathfrak{N}_{k}\right)_{k \in K}$ of $W^{*}$-subalgebras of a $W^{*}$-algebra $\mathfrak{N}$ we denote by $\bigvee_{k \in K} \mathfrak{N}_{k}$ the $W^{*}$-subalgebra of $\mathfrak{N}$ generated by the $\left.\left(\mathfrak{N}_{k}\right)_{k \in K}\right]$. With each subset $I$ of $\mathbb{Z}$ we canonically associate the faithful normal conditional expectation $E_{I}$ of $\hat{\mathfrak{M}}$ onto $\bigvee_{k \in I} \hat{\tau}^{k}(i(\mathfrak{M}))$ respecting $\hat{\psi}$. For finite $I=\left\{k_{n}, \ldots, k_{1}\right\}$ we also write $E_{k_{n}, \ldots, k_{1}}$ instead of $E_{I}$. Note that $E_{k}=\hat{\tau}^{k} \circ E \circ \hat{\tau}^{-k}$ for $k \in \mathbb{Z}$.

The dilation $(\hat{\mathfrak{M}}, \hat{\psi}, \hat{\tau} ; i, E)$ is said to have the Markov property (M) if for any finitely many $k>k_{1}>\ldots>k_{n}$ in $\mathbb{Z}$

$$
E_{k_{n}, \ldots, k_{1}}\left(\hat{\tau}^{k}(x)\right)=E_{k_{1}}\left(\hat{\tau}^{k}(x)\right) \text { for all } x \in i(\mathfrak{M}) .
$$

Then $(\hat{\mathfrak{M}}, \hat{\psi}, \hat{\tau} ; i, E)$ is also called a Markov dilation. We remark that the Markov property $(\mathrm{M})$ implies a non-commutative Feynman-Kac formula as in [4, p. 84]. In the commutative theory of dilations it is known (see e.g. [7]) that a Markov property is indispensible if one wants to establish relations between the mixing properties of a dissipative dynamical system and those of its minimal dilation.

In the following we will be concerned with a mixing property of a dilation which is defined as a natural $W^{*}$-algebraic analogue of the classical notion of a $K$-system [10].

Let $(\mathfrak{M}, \psi, \tau)$ be a conservative dynamical system. If there is an expanding subalgebra $\mathfrak{M}^{>}$of $\mathfrak{M}$ satisfying the following four conditions

(K1) $\mathfrak{M}^{>} \subseteq \tau^{n}\left(\mathfrak{M}^{>}\right)$for all $n \in \mathbb{N}$,

(K2) $\bigvee \tau^{n}\left(\mathfrak{M}^{>}\right)=\mathfrak{M}$,

(K3) $\bigcap_{n \in \mathbb{N}}^{n \in \mathbb{N}} \tau^{-n}\left(\mathfrak{M}^{>}\right)=\mathbb{C} \mathbb{1}$

(K4) there is a faithful normal conditional expectation of $\mathfrak{M}$ onto $\mathfrak{M}^{>}$ respecting $\psi$,

then $\left(\mathfrak{M}, \psi, \tau ; \mathfrak{M}^{>}\right)$is called a generalized $K$-system (cf. [2]). In addition, a generalized $K$-system $\left(\mathfrak{M}, \psi, \tau ; \mathfrak{M}^{>}\right)$is called weakly reversible [2], if $\bigvee_{n \in \mathbb{N}} \tau^{-n}(\mathfrak{M}>c)=\mathfrak{M}$. (Here and henceforth for any $W^{*}$-subalgebra $\mathfrak{N}$ of a $W^{*}$-algebra $\mathfrak{M} \in \mathbb{N}$ we denote by $\mathfrak{N}^{\complement}$ the relative commutant of $\mathfrak{N}$ in $\mathfrak{M}$, i.e. $\mathfrak{N}^{\complement}:=\{a \in \mathfrak{M}: a b=b a$ for all $b \in \mathfrak{N}\}$.) All generalized $K$-systems known so far $[2,4]$ are weakly reversible.

The purpose of the present article is to discuss the concepts introduced above for the class of those dynamical systems which arise as solutions of a standard Bloch equation for a $\frac{1}{2}$-spin [5].

To describe these dissipative dynamical systems let us denote by $\mathfrak{A}$ the $W^{*}$-algebra of all $2 \times 2$ matrices. Putting $a:=\left(\begin{array}{ll}0 & 0 \\ 1 & 0\end{array}\right) \in \mathfrak{U}$ the set $\left\{\mathbb{1}, a, a^{*}, a^{*} a\right\}$ forms a basis of $\mathfrak{A}$. On $\mathfrak{A}$ we consider a fixed faithful non-tracial state $\varphi$ given by $\varphi(a)=0, \varphi\left(a^{*} a\right)=\eta$ for a certain $0<\eta<1$. Emch and Varilly [5] have shown that for a continuous dynamical system $\left(\mathfrak{H}, \varphi, e^{t L}\right)$ with $\eta \neq \frac{1}{2}$ to admit a dilation it is necessary that the generator $L$ satisfies the following Bloch equations:

$$
L(a)=-(\lambda+i \omega) a, \quad L\left(a^{*} a-\eta \mathbb{1}\right)=-\mu\left(a^{*} a-\eta \mathbb{1}\right),
$$


where $\omega, \lambda, \mu$ are any real numbers satisfying $0 \leqq \mu \leqq 2 \lambda$. Integrating (B) yields the Bloch evolution

$$
\begin{gathered}
e^{t L}(a)=e^{-(\lambda+i \omega) t} a, \\
e^{t L}\left(a^{*} a-\eta \mathbb{1}\right)=e^{-\mu t}\left(a^{*} a-\eta \mathbb{1}\right), \quad t \in \mathbb{R}_{+} .
\end{gathered}
$$

The Eqs. (B) are easily seen to be so-called standard Bloch equations. Therefore the Bloch evolution may be interpreted as describing the relaxation of a spin- $\frac{1}{2}-$ particle at finite temperature in a magnetic field. For details and the resulting physical interpretation of the constants $\eta, \omega, \lambda, \mu$ we refer to [5].

In the following we restrict our consideration to the semigroup generated by $\gamma:=e^{L}$. Clearly, as far as ergodic theory is concerned, this does not make an essential difference. Especially it is obvious that $\gamma^{n}(x) \rightarrow \varphi(x) \cdot \mathbb{1}$ for all $x \in \mathfrak{U}$ as $n \rightarrow \infty$, i.e. $\gamma$ is strongly mixing.

For constructing dilations for $(\mathfrak{H}, \varphi, \gamma)$ it seems convenient to decompose $\gamma$ as a product $\gamma=\gamma_{1} \cdot \gamma_{2}$ of completely positive identity preserving operators $\gamma_{1}, \gamma_{2}$ on $\mathfrak{A}$, each leaving $\varphi$ invariant, namely: $\gamma_{1}$ is given by

$$
\gamma_{1}(a)=e^{-\mu / 2} a, \quad \gamma_{1}\left(a^{*} a-\eta \mathbb{1}\right)=e^{-\mu}\left(a^{*} a-\eta \mathbb{1}\right)
$$

and $\gamma_{2}$ by

$$
\gamma_{2}(a)=e^{-(\lambda-\mu / 2+i \omega)} a, \quad \gamma_{2}\left(a^{*} a\right)=a^{*} a .
$$

$\gamma_{1}$ will be called the quasifree part of $\gamma$ (cf. 2.1).

While a Markov dilation of the quasifree part was known for some time ([6]), Varilly [13] has found a minimal dilation for the whole dynamical system $(\mathfrak{A}, \varphi, \gamma)$. It is remarkable that this dilation does not inherit any ergodicity from $(\mathfrak{U}, \varphi, \gamma)$. However, as it also violates the Markov property (M) (see Sect. 2), Varilly's result should not be regarded as a final answer to the question raised by Emch [3] whether mixing properties of a dissipative dynamical system are always inherited by a minimal dilation.

In Sect. 2 we review the well known quasifree dilation in a discrete form and show that it leads to a generalized $K$-system which is not weakly reversible. For a discrete version of Varilly's dilation we demonstrate in Sect. 3 that the Markov property (M) fails. A new minimal dilation of $(\mathfrak{H}, \varphi, \gamma)$ which does satisfy the Markov property $(\mathrm{M})$ is constructed in Sect. 4. It presents the first example of a Markov dilation for a non-commutative dynamical system which is not equivalent to a quasifree evolution. Furthermore the dilation satisfies the axioms of a generalized $K$-system.

\section{The Quasifree Dilation and its Mixing Properties}

If we regard $\mathfrak{U}$ in the canonical way as the CAR algebra over the one-dimensional Hilbert space $\mathbb{C}$, then $\varphi$ appears as the quasifree state corresponding to $\eta \in] 0,1\left[\subseteq \mathbb{R}\right.$, and $\gamma_{1}$ appears as the quasifree completely positive operator induced via $\varphi$ by the contraction

$$
T: \mathbb{C} \rightarrow \mathbb{C}, z \mapsto \delta \cdot z \quad \text { with } \quad \delta:=e^{-\mu / 2} \quad \text { (see [6]). }
$$


To construct a minimal Sz.-Nagy dilation of $T$, put $\mathscr{H}:=L^{2}([0,2 \pi])$ with Lebesgue measure on $[0,2 \pi]$ and

$$
f_{1}(x):=\left(\frac{1}{2 \pi} \cdot \frac{1-\delta^{2}}{1-2 \delta \cdot \cos x+\delta^{2}}\right)^{1 / 2}, \quad x \in[0,2 \pi] .
$$

Then $f_{1}$ is a positive bounded function in $L^{2}([0,2 \pi])$. If we define a unitary operator $U$ on the Hilbert space $\mathscr{H}$ by

$$
(U g)(x)=e^{i x} g(x) \text { for } g \in L^{2}([0,2 \pi]), \quad x \in[0,2 \pi],
$$

then $U$ satisfies $\left\langle U^{n} f_{1} \mid f_{1}\right\rangle=\left\langle U^{n} 1 \mid f_{1}^{2}\right\rangle=T^{n} 1$ for $n \in \mathbb{N}$, and we have obtained a minimal Sz.-Nagy dilation of $T$ ([12]).

Now the quasifree dilation scheme of [6] can be applied to $\left(\mathfrak{H}, \varphi, \gamma_{1}\right)$ :

Let $A(\mathscr{H})$ be the CAR algebra over $\mathscr{H}$ and denote by $\psi_{1}$ the gauge invariant quasifree state on $A(\mathscr{H})$ determined by $\psi_{1}\left(a^{*}(f) a(g)\right)=\eta\langle g \mid f\rangle$ for $f, g \in \mathscr{H}$. Define $\mathfrak{C}$ as the weak closure of $A(\mathscr{H})$ in the GNS-representation with respect to $\psi_{1}$. Then $\mathbb{C}$ is the hyperfinite factor of type $\mathrm{III}_{\eta /(1-\eta)}$ (respectively $\mathrm{II}_{1}, \mathrm{III}_{(1-\eta) / \eta}$ ) if $0<\eta<\frac{1}{2}$ (respectively $\eta=\frac{1}{2}, \frac{1}{2}<\eta<1$ ). The state $\psi_{1}$ extends to a faithful normal state on $\mathfrak{C}$ denoted by $\psi_{1}$, too. A quasifree automorphism $\alpha_{1}$ on $\mathbb{C}_{1}$ is determined uniquely by $\alpha_{1}(a(g)):=a(U g)$ for $g \in \mathscr{H}$. For the required injection take $i_{1}: \mathfrak{U} \rightarrow \mathfrak{C}, a \mapsto a\left(f_{1}\right)$ and for the corresponding projection the uniquely determined normal conditional expectation $R$ from $\mathbb{C}$ onto $i_{1}(\mathfrak{U})$ respecting $\psi_{1}$.

Then $\left(\mathfrak{C}, \psi_{1}, \alpha_{1} ; i_{1}, R\right)$ is a minimal Markov dilation of $\left(\mathfrak{A}, \varphi, \gamma_{1}\right)$.

An analysis of the mixing properties of quasifree dilations over the CAR seems not to be available in the literature. For quasifree dilations over the CCR an ergodic theory can be found in [4]. There on p. 83 the authors remark that the corresponding theory for Fermi systems can be obtained in an analogous way "with the proviso that one works with the observable-algebra rather than the fieldalgebra." In the proof of the following theorem we develop a method which applies to quasifree dilations over the full CAR algebra, i.e. the field algebra. For a systematic account we refer to [11].

Theorem. With the expanding subalgebra $\mathfrak{C}^{>}:=\bigvee_{n \in \mathbb{N}} \alpha_{1}^{-n} \cdot i_{1}(\mathfrak{U})\left(\mathfrak{C}, \psi_{1}, \alpha_{1} ; \mathfrak{C}^{>}\right)$is a generalized K-system.

Proof. While properties (K 1), (K 2), and (K4) are easily checked, for (K 3) we employ some ideas of the theory of quasilocal algebras (cf. [1], in particular Theorem 2.6.5). For $k \in \mathbb{Z}$ let $\mathscr{H}(-\infty, k)$ be the closed linear hull of $\left\{U^{n} f_{1}: n \in \mathbb{Z}\right.$, $n<k\}$ in $\mathscr{H}$, and $\mathscr{H}(k,+\infty)$ its orthogonal complement in $\mathscr{H}$. Then $\mathfrak{C}(-\infty, k):=\alpha_{1}^{k}\left(\mathfrak{C}^{>}\right)$is the $W^{*}$-subalgebra of $\mathfrak{C}$ generated by $\{a(f)$ : $f \in \mathscr{H}(-\infty, k)\} \cong A(\mathscr{H})$. Correspondingly let $\mathfrak{C}(k,+\infty)$ be the $W^{*}$-subalgebra of $\mathfrak{C}$ generated by $\{a(g): g \in \mathscr{H}(k,+\infty)\} \cong A(\mathscr{H})$ and put

$$
\mathfrak{C}(m, n):=\mathfrak{C}(-\infty, n) \cap \mathfrak{C}(m,+\infty) \text { for } m, n \in \mathbb{Z}, m \leqq n .
$$

Subalgebras corresponding this way to disjoint subsets of $\mathbb{Z}$ satisfy special commutation relations arising from the CAR. These commutation relations may be expressed by means of the automorphism $\varepsilon$ of $\mathcal{C}$ defined by $\varepsilon(a(f))=-a(f)$ for $f \in \mathscr{H}$. 
For any $z \in \mathbb{C}$ put $z^{e}:=\frac{1}{2}(z+\varepsilon(z))$ and $z^{o}:=\frac{1}{2}(z-\varepsilon(z))$, and for any subset $\mathfrak{B}$ of $\mathfrak{C}$ define $\mathfrak{B}^{e}:=\left\{z \in \mathfrak{B}: z=z^{e}\right\}$ and $\mathfrak{B}^{o}:=\left\{z \in \mathfrak{B}: z=z^{o}\right\}$. Now, if $-\infty \leqq m \leqq n \leqq k$ $\leqq l \leqq+\infty$, we have

$$
\begin{array}{lll}
y z=z y & \text { for all } & y \in \mathfrak{C}(m, n)^{e}, z \in \mathfrak{C}(k, l), \\
y z=-z y & \text { for all } & y \in \mathbb{C}(m, n)^{o}, z \in \mathbb{C}(k, l)^{o} .
\end{array}
$$

Given a self-adjoint $x \in \bigcap_{n} \mathfrak{C}(-\infty,-n),\|x\|=1$, we want to conclude from $(* *)$ that $x^{o}=0$.

Observe that for any $n \in \mathbb{N}, \bigcup_{k>n} \mathfrak{C}(-k,-n)$ is an ultrastrongly dense *-subalgebra of $\mathfrak{C}(-\infty,-n)$. Hence by Kaplansky's density theorem we can choose a sequence of self-adjoint $x_{n} \in \mathfrak{C},\left\|x_{n}\right\| \leqq 1$, which converges to $x$ ultrastrongly and in addition satisfies the following property: For each $n \in \mathbb{N}$ there is a $k_{n}>n$ such that $x_{n} \in \mathcal{C}\left(-k_{n},-n\right)$. Then by the continuity of $\varepsilon: \lim _{n} x_{n}^{o}=x^{o}$ ultrastrongly. Since multiplication is jointly ultrastrongly continuous on the unit ball of $\mathfrak{C}$, we conclude $\left(x^{o}\right)^{2}=\lim _{n} \lim _{m} x_{n}^{o} x_{m}^{o}$. Now for $n \in \mathbb{N}$ we have $k_{n}>n$ such that $x^{0} \in \mathfrak{C}\left(-k_{n},-n\right)^{o}$, and so for any $m>k_{n}$ we get $x_{n}^{o} x_{m}^{o}=-x_{m}^{o} x_{n}^{o}$ by property $(* *)$. Therefore

$$
\begin{aligned}
\left(x^{o}\right)^{2} & =\lim _{n}\left(-\lim _{m} x_{m}^{o} x_{n}^{o}\right) \\
& =-\lim _{n} x^{o} x_{n}^{o} \\
& =-\left(x^{o}\right)^{2}
\end{aligned}
$$

which implies $x^{o}=0$. This means $\bigcap_{m \in \mathbb{N}} \mathfrak{C}(-\infty,-m)=\bigcap_{m \in \mathbb{N}} \mathfrak{C}(-\infty,-n)^{e}$.

Now property $(*)$ gives $\mathbb{C}(-\infty,-n)^{e} \cong \mathfrak{C}(-n,+\infty)^{\complement}$ for all $n \in \mathbb{N}$. Since $\bigcap_{n} \mathfrak{C}(-n,+\infty)^{\mathfrak{C}}$ is the center of $\mathfrak{C}$ and $\mathfrak{C}$ is a factor, we have proved $\bigcap_{n}^{n} \mathfrak{C}(-\infty,-n) \subseteq \mathbb{C} \mathbb{1}$ which is property $(\mathrm{K} 3)$.

Generalized $K$-systems built over the CAR algebra like $\left(\mathfrak{C}, \psi_{1}, \alpha_{1} ; \mathfrak{C}^{>}\right)$seem to be the first examples of generalized $K$-systems which are not weakly reversible.

Proposition. $\left(\mathfrak{C}, \psi_{1}, \alpha_{1} ; \mathfrak{C}^{>}\right)$is not weakly reversible.

Proof. Suppose to the contrary that $\bigcup_{n} \alpha_{1}^{-n}\left(\mathfrak{C}^{>c}\right)$ is ultrastrongly dense in $\mathfrak{C}$. Define $p(x):=\psi_{1}\left(x^{*} x\right)^{1 / 2}$ and $\left[x_{1}, x_{2}\right]:=x_{1} x_{2}-x_{2} x_{1}$ for all $x, x_{1}, x_{2} \in \mathfrak{C}$ and put $y:=a\left(f_{1}\right)=i_{1}(a)$. We want to estimate $p\left(\left[\alpha_{1}^{-n}(y), y\right]\right)$. As on bounded sets the ultrastrong topology coincides with the topology generated by $p$, for $\eta=\varphi\left(a^{*} a\right)>0$ there is an $\varepsilon>0$ such that for any $x \in \mathbb{C},\|x\| \leqq 2, p(x)<\varepsilon$ implies both $p(x)<\eta / 2$ and $p(x y)<\eta / 2$. Now by Kaplansky's density theorem choose $m \in \mathbb{N}$ and $z \in \alpha_{1}^{-m}\left(\mathfrak{C}^{>c}\right)$, $\|z\| \leqq 1$, such that $p(y-z)<\varepsilon$. This allows the estimate:

$$
\begin{aligned}
p\left(\left[\alpha_{1}^{-n}(y), y\right]\right) \leqq & p\left(\left[\alpha_{1}^{-n}(y), z\right]\right)+p\left(\left[\alpha_{1}^{-n}(y), y-z\right]\right) \\
\leqq & \left\|\alpha_{1}^{-n}(y)\right\| p(y-z)+p\left(\alpha_{1}^{n}(y-z) y\right) \\
& \text { for } n>m \text { because then }\left[\alpha_{1}^{-n}(y), z\right]=0
\end{aligned}
$$


Since $p\left(\alpha_{1}^{n}(y-z)\right)=p(y-z)<\varepsilon$ and $\|y-z\| \leqq 2$ we obtain $p\left(\left[\alpha_{1}^{-n}(y), y\right]\right)<\eta$. However, this contradicts

$$
p\left(\left[\alpha_{1}^{-n}(y), y\right]\right)=2 \eta\left(1-e^{-\mu \cdot n}\right)^{1 / 2} \rightarrow 2 \eta \quad \text { for } n \rightarrow \infty .
$$

\section{Varilly's Dilation and the Violation of the Markov Property}

On the basis of the quasifree dilation Varilly [13] has proposed a minimal dilation of the whole dynamical system $(\mathfrak{A}, \varphi, \gamma)$. In a discrete version it takes the following form:

Define $\varrho:=e^{-(\lambda-\mu / 2)}$ and $f_{2} \in L^{1}([0,2 \pi])$ by

$$
f_{2}(x):=\frac{1}{2 \pi} \frac{1-\varrho^{2}}{1-2 \varrho \cdot \cos (x+\omega)+\varrho^{2}} \quad \text { for } \quad x \in[0,2 \pi] \text {. }
$$

Then $\psi_{2}(g):=\int_{0}^{2 \pi} g(x) f_{2}(x) d x$ for $g \in L^{\infty}([0,2 \pi])$ defines a faithful normal state $\psi_{2}$ on $L^{\infty}([0,2 \pi])$. Now put $\mathfrak{N}:=\mathbb{C} \otimes L^{\infty}([0,2 \pi]), \hat{\psi}:=\psi_{1} \otimes \psi_{2}$, define an automorphism $\hat{\gamma}$ of $\mathfrak{N}$ by $\hat{\gamma}(a(f) \otimes g):=a(U f) \otimes U g$ for $f \in \mathscr{H}, g \in L^{\infty}([0,2 \pi])$, an embedding $j: \mathfrak{A} \rightarrow \mathfrak{N}$ by $j(a):=a\left(f_{1}\right) \otimes \mathbb{1}$, and finally a faithful normal conditional expectation $\bar{P}$ of $\mathfrak{N}$ onto $j(\mathfrak{U})$ by $\bar{P}(x \otimes g):=\psi_{2}(g) R(x) \otimes \mathbb{1}$ for $x \in \mathfrak{C}, g \in L^{\infty}([0,2 \pi])$.

Theorem (Varilly [13]). ( $\mathfrak{N}, \hat{\psi}, \hat{\gamma} ; j, \bar{P})$ is a minimal dilation of $(\mathfrak{A}, \varphi, \gamma)$.

However, as pointed out by Varilly [13], this dilation does not show any mixing properties in spite of the strong mixing of $(\mathfrak{A}, \varphi, \gamma)$. In fact, the center $\mathbb{1} \otimes L^{\infty}([0,2 \pi])$ of $\mathfrak{N}$ is pointwise fixed under the automorphism $\hat{\gamma}$.

As already remarked in Sect. 1 we can hope for a relation between the mixing properties of $(\mathfrak{A}, \varphi, \gamma)$ and those of its minimal dilation only, if a Markov property holds.

Lemma. The Varilly dilation $(\mathfrak{N}, \hat{\psi}, \hat{\gamma} ; j, \bar{P})$ does not possess the Markov property (M).

Proof. We show that $\bar{P}_{1}\left(\hat{\gamma}^{2}(j(a))\right) \neq \bar{P}_{0,1}\left(\hat{\gamma}^{2}(j(a))\right)$ for $a=\left(\begin{array}{ll}0 & 0 \\ 1 & 0\end{array}\right)$. By the dilation property we obtain

$$
\begin{aligned}
\bar{P}_{1}\left(\hat{\gamma}^{2}(j(a))\right) & =\hat{\gamma}(j(\gamma(a)))=e^{-(\lambda+i \omega)} \hat{\gamma}(j(a)) \\
& =e^{-(\lambda+i \omega)} a\left(U f_{1}\right) \otimes U
\end{aligned}
$$

On the other hand, by the anti-commutation relations,

$$
j(a)^{*} \hat{\gamma}(j(a))+\hat{\gamma}(j(a)) j(a)^{*}=e^{-\frac{\mu}{2}} \mathbb{1} \otimes U,
$$

and therefore

$$
\mathbb{1} \otimes L^{\infty}([0,2 \pi]) \subseteq \bar{P}_{0,1}(\mathfrak{N}) .
$$

Furthermore $\left(a\left(U f_{1}\right) \otimes U\right) \cdot\left(\mathbb{1} \otimes U^{*}\right)=a\left(U f_{1}\right) \otimes \mathbb{1} \in \bar{P}_{0,1}(\mathfrak{N})$. Hence $\bar{P}_{0,1}(\mathfrak{N})=\mathbb{C}_{0,1}$ $\otimes L^{\infty}([0,2 \pi])$, where $\mathfrak{C}_{0,1}$ is the subalgebra of $\mathbb{C}$ generated by $a\left(f_{1}\right)$ and $a\left(U f_{1}\right)$. Since $\bar{P}_{0,1}(\mathfrak{C} \otimes \mathbb{1})=\mathfrak{C}_{0,1} \otimes \mathbb{1}$, we obtain 


$$
\begin{aligned}
\bar{P}_{0,1}\left(\hat{\gamma}^{2}(j(a))\right) & =\bar{P}_{0,1}\left(a\left(U^{2} f_{1}\right) \otimes U^{2}\right) \\
& =\bar{P}_{0,1}\left(a\left(U^{2} f_{1}\right) \otimes \mathbb{1}\right) \cdot \mathbb{1} \otimes U^{2} \\
& =R_{0,1}\left(a\left(U^{2} f_{1}\right)\right) \otimes \mathbb{1} \cdot \mathbb{1} \otimes U^{2} \\
& =R_{1}\left(a\left(U^{2} f_{1}\right)\right) \otimes \mathbb{1} \cdot \mathbb{1} \otimes U^{2} \\
& =e^{-\frac{\mu}{2} a\left(U f_{1}\right) \otimes U^{2},}
\end{aligned}
$$

where the last but one equation follows from the Markov property of $\left(\mathfrak{C}, \psi_{1}, \alpha_{1} ; i_{1}, R\right)$.

\section{A New Dilation of the Bloch Evolution}

In this paragraph we first elaborate the general scheme of [8] for constructing a Markov dilation of the dynamical system $\left(\mathfrak{A}, \varphi, \gamma_{2}\right)$. In the next step we combine it in an appropriate manner with the dilation of $\left(\mathfrak{A}, \varphi, \gamma_{1}\right)$ described in Sect. 2. This gives us a new minimal conservative dilation for the dynamical system $(\mathfrak{A}, \varphi, \gamma)$ which satisfies both the Markov property (M) and the axioms of a generalized $K$-system.

Let $(Y, \vartheta)$ be the measure space with $Y:=\{0,1\}, \vartheta(\{0\})=\vartheta(\{1\})=1 / 2$, $(X, v):=(Y, \vartheta)^{\mathbb{Z} *}$ with $\mathbb{Z}^{*}:=\mathbb{Z} \backslash\{0\}$. An element $\xi \in X$ may be identified with a $\{0,1\}$ valued function $\xi(n)$ on $\mathbb{Z}^{*}$. Put $\mathfrak{B}:=L^{\infty}(X, v)$ and denote by $\varphi_{2}$ the faithful normal state on $\mathfrak{B}$ given by $\varphi_{2}(f):=\int_{X} f(\xi) d v$. We define $e_{0}$ and $e_{1} \in \mathfrak{B}$ as the functions

$$
e_{0}(\xi):=\left\{\begin{array}{lll}
1 & \text { if } & \xi(1)=0 \\
0 & \text { if } & \xi(1)=1
\end{array}, \quad e_{1}(\xi):=\left\{\begin{array}{lll}
1 & \text { if } & \xi(1)=1 \\
0 & \text { if } & \xi(1)=0
\end{array} .\right.\right.
$$

Then $e_{0}$ and $e_{1}$ are projections with $e_{0}+e_{1}=\mathbb{1}, \varphi_{2}\left(e_{0}\right)=\varphi_{2}\left(e_{1}\right)=1 / 2$.

For $\varrho:=e^{-\left(\lambda-\frac{\mu}{2}\right)}$, we define the unitaries

$$
v_{0}:=\left(\begin{array}{cc}
1 & 0 \\
0 & e^{i \omega}\left(\varrho-i \sqrt{1-\varrho^{2}}\right)
\end{array}\right), \quad v_{1}:=\left(\begin{array}{cc}
1 & 0 \\
0 & e^{i \omega}\left(\varrho+i \sqrt{1-\varrho^{2}}\right)
\end{array}\right)
$$

in $\mathfrak{A}$. Then $V:=v_{0} \otimes e_{0}+v_{1} \otimes e_{1}$ is a unitary element in $\mathfrak{A} \otimes \mathfrak{\otimes}$. Furthermore we note that for $x \in \mathfrak{A} \frac{1}{2}\left(v_{0}^{*} x v_{0}+v_{1}^{*} x v_{1}\right)=\gamma_{2}(x)$. The right shift

$$
s: X \rightarrow X,(s \xi)(n):=\left\{\begin{array}{lll}
\xi(n-1) & \text { if } & n \neq 1 \\
\xi(-1) & \text { if } & n=1
\end{array}\right.
$$

is a measure preserving transformation on $(X, v)$ which induces a $*$-automorphism $\bar{\sigma}$ on $\mathfrak{B}$. Now we get the ${ }^{*}$-automorphisms $\sigma:=\operatorname{Id} \otimes \bar{\sigma}$ on $\mathfrak{A} \otimes \bar{\otimes} \mathfrak{B}$ and

$$
\alpha_{2}: \mathfrak{A} \bar{\otimes} \mathfrak{B} \rightarrow \mathfrak{A} \bar{\otimes} \mathfrak{B}, \quad x \mapsto V^{*} \cdot \sigma(x) \cdot V .
$$

Finally we put

$$
\begin{gathered}
i_{2}: \mathfrak{U} \rightarrow \mathfrak{U} \bar{\otimes} \mathfrak{B}, \quad x \mapsto x \otimes \mathbb{1} \\
Q: \mathfrak{U} \bar{\otimes} \mathfrak{B} \rightarrow \mathfrak{U} \otimes \mathbb{1}, \quad x \otimes y \mapsto \varphi_{2}(y) \cdot x \otimes \mathbb{1} .
\end{gathered}
$$


Proposition. ( $\left(\mathfrak{A} \otimes \mathfrak{B}, \varphi \otimes \varphi_{2}, \alpha_{2} ; i_{2}, Q\right)$ is a minimal dilation of the dynamical system $\left(\mathfrak{A}, \gamma_{2}, \varphi\right)$ and satisfies the Markov property $(\mathrm{M})$.

Before beginning with the proof we fix some more notation: If $I \subseteq \mathbb{Z}^{*}$,

$$
\mathfrak{B}_{I}:=\{f \in \mathfrak{B}: f(\xi)=f(\eta) \text { if } \xi(i)=\eta(i) \text { for all } i \in I\}
$$

is a subalgebra of $\mathfrak{B}$ which is canonically isomorphic to $L^{\infty}\left((Y, \vartheta)^{I}\right)$. Denote by $\bar{Q}_{I}$ the conditional expectation from $\mathfrak{U} \bar{\otimes} \mathfrak{B}$ onto $\mathfrak{A} \otimes \mathfrak{B}_{I}$ respecting $\varphi \otimes \varphi_{2}$, and observe that $\bar{Q}_{I}(\mathfrak{H} \bar{\otimes} \mathfrak{B}) \supseteqq Q_{I \cup\{0\}}(\mathfrak{U} \otimes \bar{\otimes})$, where $Q_{I \cup\{0\}}$ is defined as in Sect. 1 .

Proof. For $x \in \mathfrak{A}: \varphi(x)=\varphi\left(v_{0}^{*} \cdot x \cdot v_{0}\right)=\varphi\left(v_{1}^{*} \cdot x \cdot v_{1}\right)$, hence $\alpha_{2}$ leaves $\varphi \otimes \varphi_{2}$ invariant. Next we get for $x \in \mathfrak{A}$ and for all $I=\{-k, \ldots,-1\} \cong-\mathbb{N}$ :

$$
\begin{aligned}
\bar{Q}_{I}\left(\alpha_{2}\left(i_{2}(x)\right)\right) & =\bar{Q}_{I}\left(\alpha_{2}(x \otimes \mathbb{1})\right) \\
& =\bar{Q}_{I}\left(V^{*} \cdot \sigma(x \otimes \mathbb{1}) \cdot V\right) \\
& =\bar{Q}_{I}\left(V^{*} \cdot x \otimes \mathbb{1} \cdot V\right) \\
& =\bar{Q}_{I}\left(v_{0}^{*} \cdot x \cdot v_{0} \otimes e_{0}+v_{1}^{*} \cdot x \cdot v_{1} \otimes e_{1}\right) \\
& =v_{0}^{*} \cdot x \cdot v_{0} \otimes \frac{1}{2} \cdot \mathbb{1}+v_{1}^{*} \cdot x \cdot v_{1} \otimes \frac{1}{2} \cdot \mathbb{1} \quad\left(e_{0}, e_{1} \in \mathfrak{B}_{1}\right) \\
& =\frac{1}{2}\left(v_{0}^{*} \cdot x \cdot v_{0}+v_{1}^{*} \cdot x \cdot v_{1}\right) \otimes \mathbb{1} \\
& =\gamma_{2}(x) \otimes \mathbb{1} \\
& =i_{2}\left(\gamma_{2}(x)\right) \\
& =Q\left(\alpha_{2}\left(i_{2}(x)\right)\right),
\end{aligned}
$$

which implies the Markov property (M) for our dilation.

By noting that $Q=Q \cdot \bar{Q}_{1} \ldots \bar{Q}_{1,2, \ldots, n-1}(n \in \mathbb{N})$ and $\bar{Q}_{1, \ldots, k}\left(\sigma^{m}\left(V^{*}\right) \cdot y \cdot \sigma^{m}(V)\right)$ $=\sigma^{m}\left(V^{*}\right) \cdot \bar{Q}_{1, \ldots, k}(y) \cdot \sigma^{m}(V)(k-1 \geqq m \geqq 0, y \in \mathfrak{A} \otimes \mathfrak{B})$, we obtain

$$
\begin{aligned}
Q \alpha_{2}^{n}\left(i_{2}(x)\right)= & Q\left[V^{*} \cdot \sigma\left[V^{*} \cdot \sigma\left[V^{*} \ldots \sigma\left[V^{*} \cdot \sigma(x \otimes \mathbb{1}) \cdot V\right] \ldots V\right] \cdot V\right] \cdot V\right] \\
= & Q\left[V^{*} \cdot \sigma\left(V^{*}\right) \cdot \sigma^{2}\left(V^{*}\right) \ldots \sigma^{n-1}\left(V^{*} \cdot(x \otimes \mathbb{1}) \cdot V\right) \ldots \sigma^{2}(V) \cdot \sigma(V) \cdot V\right] \\
= & Q\left[V ^ { * } \cdot \overline { Q } _ { 1 } \left[\sigma ( V ^ { * } ) \cdot \overline { Q } _ { 1 , 2 } \left[\sigma^{2}\left(V^{*}\right) \ldots \bar{Q}_{1, \ldots, n-1}\left[\sigma^{n-1}\left(V^{*} \cdot(x \otimes \mathbb{1}) \cdot V\right)\right] \ldots\right.\right.\right. \\
& \left.\left.\left.\ldots \sigma^{2}(V)\right] \cdot \sigma(V)\right] \cdot V\right] \\
= & Q\left[V^{*} \cdot \bar{Q}_{1}\left[\sigma\left(V^{*}\right) \cdot \bar{Q}_{1,2}\left[\sigma^{2}\left(V^{*}\right) \ldots \sigma^{n-1}\left(\gamma_{2}(x) \otimes \mathbb{1}\right) \ldots \sigma^{2}(V)\right] \cdot \sigma(V)\right] \cdot V\right] \\
& \vdots \\
= & Q\left[V^{*} \cdot\left(\gamma_{2}^{n-1}(x) \otimes \mathbb{1}\right) \cdot V\right] \\
= & \gamma_{2}^{n}(x) \otimes \mathbb{1} \\
= & i_{2}\left(\gamma_{2}^{n}(x)\right) \quad(x \in \mathfrak{U}),
\end{aligned}
$$

which proves the dilation property with the aid of the Markov property (M) proved above.

It remains to show minimality: One easily checks that $i_{2}(\mathfrak{A}) \vee \alpha_{2}\left(i_{2}(\mathfrak{U})\right)$ $=\mathfrak{U} \bar{\otimes} \mathfrak{B}_{1}$. Furthermore 
and by

$$
\begin{aligned}
\alpha_{2}\left(i_{2}(\mathfrak{I})\right) \vee \alpha_{2}^{2}\left(i_{2}(\mathfrak{I})\right) & =\alpha_{2}\left[i_{2}(\mathfrak{U}) \vee \alpha_{2}\left(i_{2}(\mathfrak{I})\right)\right] \\
& =\alpha_{2}\left(\mathfrak{I} \otimes \mathfrak{B}_{1}\right) \\
& \supseteqq \alpha_{2}\left(\mathbb{1} \otimes \mathfrak{B}_{1}\right) \\
& =\mathbb{1} \otimes \mathfrak{B}_{2},
\end{aligned}
$$

we see that

$$
\mathfrak{U} \bar{\otimes} \mathfrak{B}_{1} \vee \mathbb{1} \otimes \mathfrak{B}_{2}=\mathfrak{U} \bar{\otimes}\left(\mathfrak{B}_{1} \vee \mathfrak{B}_{2}\right)=\mathfrak{U} \bar{\otimes} \mathfrak{B}_{1,2}
$$

$$
i_{2}(\mathfrak{U}) \vee \alpha_{2}\left(i_{2}(\mathfrak{U})\right) \vee \alpha_{2}^{2}\left(i_{2}(\mathfrak{U})\right)=\mathfrak{U} \bar{\otimes} \mathfrak{B}_{1,2} .
$$

Continuing this procedure yields

$$
\bigvee_{n \in \mathbb{Z}} \alpha_{2}^{n}\left(i_{2}(\mathfrak{A})\right)=\mathfrak{U} \bar{\otimes} \mathfrak{B}
$$

which proves minimality.

Now we are in a position to define the announced conservative dilation for the dissipative dynamical system $(\mathfrak{A}, \varphi, \gamma)$. For $g \in \mathscr{H}$ denote by $\mathfrak{A}(g)$ the subalgebra of $\mathfrak{C}$ generated by $a(g)$ and if $\|g\|=1$, denote by $\pi_{g}$ the uniquely determined *-isomorphism of $\mathfrak{U}$ into $\mathfrak{C}$ such that $\pi_{g}(\mathfrak{A})=\mathfrak{A}(g)$ and $\pi_{g}(a)=\pi_{g}\left(\begin{array}{ll}0 & 0 \\ 1 & 0\end{array}\right)=a(g)$. Note that $\alpha_{1}\left(\pi_{g}(x)\right)=\pi_{U g}(x)$ for $x \in \mathfrak{A}$. On $\hat{\mathfrak{A}}:=\mathfrak{C} \otimes \mathfrak{B}$ we now define a *-automorphism $\hat{\alpha}$ by

$$
\hat{\alpha}:=\alpha_{1} \otimes \operatorname{Id} \cdot\left(\sigma_{t_{0}}^{\psi_{1}} \otimes \operatorname{Id} \uparrow_{\mathfrak{c} \otimes e_{0} \mathfrak{B}}+\sigma_{t_{1}}^{\psi_{1}} \otimes \operatorname{Id} \uparrow_{\mathfrak{C} \otimes e_{1} \mathfrak{B}}\right) \cdot \operatorname{Id} \otimes \bar{\sigma},
$$

where $\left(\sigma_{t}^{\psi_{1}}\right)_{t \in \mathbb{R}}$ denotes the modular automorphism group on $\mathfrak{C}$ corresponding to $\psi_{1}$, and $t_{0}, t_{1}$ satisfy

$$
\begin{aligned}
& \left(\frac{\eta}{1-\eta}\right)^{i t_{0}}=e^{i \omega}\left(\varrho-i \sqrt{1-\varrho^{2}}\right) \\
& \left(\frac{\eta}{1-\eta}\right)^{i t_{1}}=e^{i \omega}\left(\varrho+i \sqrt{1-\varrho^{2}}\right) .
\end{aligned}
$$

For $x \in \mathfrak{U}, h \in \mathfrak{B}$ this definition yields

$$
\begin{aligned}
\hat{\alpha}\left(\pi_{g}(x) \otimes h\right) & =\hat{\alpha}\left(\left(\pi_{g} \otimes \mathrm{Id}\right)(x \otimes h)\right) \\
& =\left(\pi_{U g} \otimes \mathrm{Id}\right)\left(\alpha_{2}(x \otimes h)\right) .
\end{aligned}
$$

Furthermore we define a faithful normal state $\hat{\varphi}:=\phi_{1} \otimes \psi_{2}$ on $\hat{\mathfrak{A}}$, an injection $i: \mathfrak{U} \rightarrow \hat{\mathfrak{A}}, \quad x \mapsto \pi_{f_{1}}(x) \otimes \mathbb{1}, \quad$ and $a$ conditional expectation $\quad P: \hat{\mathfrak{U}} \rightarrow i(\mathfrak{H}), \quad x \otimes h$ $\mapsto \varphi_{2}(h) \cdot R(x) \otimes \mathbb{1}=Q \cdot(R \otimes I d)(x \otimes h)$.

Theorem. ( $\hat{\mathfrak{U}}, \hat{\varphi}, \hat{\alpha} ; i, P)$ is a minimal dilation of the dynamical system $(\mathfrak{U}, \varphi, \gamma)$.

For $I \subseteq \mathbb{Z}$ we denote by $\hat{\mathfrak{U}}_{I}\left(\right.$ respectively $\left.\mathfrak{C}_{I}\right)$ the $W^{*}$-subalgebra $P_{I}(\hat{\mathfrak{U}})$ of $\hat{\mathfrak{U}}$ [respectively $R_{I}(\mathfrak{C})$ of $\left.\mathfrak{C}\right]$.

Proof. First we show minimality. For $r:=\varrho+i \sqrt{1-\varrho^{2}}, \varrho=e^{-\left(\lambda-\frac{\mu}{2}\right)}$, and $\bar{r}$ the complex conjugate of $r$, we define $W:=r e_{0}+\bar{r} e_{1} \in \mathfrak{B}_{1}$. The unitary $\bar{\sigma}^{n-1}(W)$ generates $\mathfrak{B}_{n}(n \geqq 1)$. An easy computation shows that 


$$
\hat{\alpha}^{n}(i(a))=e^{-i n \omega} a\left(U^{n} f_{1}\right) \otimes \prod_{l=0}^{n-1} \bar{\sigma}^{l}(W) .
$$

For its anti-commutator with $i(a)^{*}$ one obtains

$$
\begin{aligned}
{\left[i(a)^{*}, \hat{\alpha}^{n}(i(a))\right]_{+} } & =e^{-i n \omega}\left[a\left(f_{1}\right)^{*}, a\left(U^{n} f_{1}\right)\right]_{+} \otimes \prod_{l=0}^{n-1} \bar{\sigma}^{l}(W) \\
& =e^{-i n \omega} e^{-n \frac{\mu}{2}} \mathbb{1} \otimes \prod_{l=0}^{n-1} \bar{\sigma}^{l}(W) \in \hat{\mathfrak{U}}_{0, \ldots, n}, \quad(n \geqq 1) .
\end{aligned}
$$

For $n=1$ it follows that $\mathbb{1} \otimes \mathfrak{B}_{1} \subseteq \hat{\mathfrak{A}}_{0,1}$. If we assume that $\mathbb{1} \otimes \mathfrak{B}_{1, \ldots, n-1}$ $\subseteq \hat{\mathfrak{U}}_{0, \ldots, n-1}$, then

$$
\left(\mathbb{1} \otimes \prod_{l=0}^{n-2} \bar{\sigma}^{l}(W)^{*}\right)\left(\mathbb{1} \otimes \prod_{l=0}^{n-1} \bar{\sigma}^{l}(W)\right)=\mathbb{1} \otimes \bar{\sigma}^{n-1}(W) \in \hat{\mathfrak{A}}_{0, \ldots, n}
$$

implies

$$
\begin{aligned}
\hat{\mathfrak{A}}_{0, \ldots, n} & \supseteqq \mathbb{1} \otimes \mathfrak{B}_{1, \ldots, n-1} \vee \mathbb{1} \otimes \mathfrak{B}_{n} \\
& =\mathbb{1} \otimes \mathfrak{B}_{1, \ldots, n} \quad(n \geqq 2) .
\end{aligned}
$$

Hence $\hat{\alpha}(i(a))\left(\mathbb{1} \otimes \prod_{l=0}^{k-1} \bar{\sigma}^{-1}(W)^{*}\right)=e^{-i k \omega} a\left(U^{k} f_{1}\right) \otimes \mathbb{1}$ with $0 \leqq k \leqq n$ is an element of $\hat{\mathfrak{Y}}_{0, \ldots, n}$, too. We conclude that $\hat{\mathfrak{U}}_{0, \ldots, n}=\mathfrak{C}_{0, \ldots, n} \bar{\otimes} \mathfrak{B}_{1, \ldots, n},(n \geqq 1)$. Combined with $\hat{\mathfrak{U}}_{0, \ldots,-n}=\mathfrak{C}_{0, \ldots,-n} \bar{\otimes} \mathfrak{B}_{1, \ldots,-n},(n \geqq 1)$, which can be obtained analogously, minimality is proved.

It remains to exhibit the dilation property: We identify $\mathfrak{A} \otimes \mathfrak{B}$ with $\pi_{f_{1}}(\mathfrak{U}) \bar{\otimes} \mathfrak{B}$, and get for any $x \in \mathfrak{U}, n \in \mathbb{N}$ :

$$
\begin{aligned}
P\left(\hat{\alpha}^{n}(i(x))\right) & =P\left[\hat{\alpha}^{n}\left(\pi_{f_{1}}(x) \otimes \mathbb{1}\right)\right] \\
& \left.=P\left[\alpha_{1}^{n} \otimes \operatorname{Id}\right)\left(\alpha_{2}^{n}\left(\pi_{f_{1}}(x) \otimes \mathbb{1}\right)\right)\right] \\
& =R \otimes \operatorname{Id}\left[\left(\alpha_{1}^{n} \otimes \operatorname{Id}\left(Q\left(\alpha_{2}^{n}\left(\pi_{f_{1}}(x) \otimes \mathbb{1}\right)\right)\right)\right)\right] \\
& =R \otimes \operatorname{Id}\left[\alpha_{1}^{n} \otimes \operatorname{Id}\left(\pi_{f_{1}}\left(\gamma_{2}^{n}(x)\right) \otimes \mathbb{1}\right)\right] \\
& =R \otimes \operatorname{Id}\left[\alpha_{1}^{n} \pi_{f_{1}}\left(\gamma_{2}^{n}(x)\right) \otimes \mathbb{1}\right] \\
& =\pi_{f_{1}}\left(\gamma_{1}^{n} \gamma_{2}^{n}(x)\right) \otimes \mathbb{1} \\
& =i\left(\gamma^{n}(x)\right) .
\end{aligned}
$$

Theorem. a) The minimal dilation ( $\hat{\mathfrak{A}}, \hat{\varphi}, \hat{\alpha} ; i, P$ ) satisfies the Markov property (M).

b) With the expanding subalgebra $\hat{\mathfrak{U}}^{>}:=\bigvee_{n \in \mathbb{N}} \hat{\alpha}^{-n} \cdot i(\mathfrak{U})\left(\hat{\mathfrak{A}}, \hat{\varphi}, \hat{\alpha} ; \hat{\mathfrak{Y}}^{>}\right)$is a generalized $K$-system, which is not weakly reversible.

The restriction to the center, $(\mathbb{1} \otimes \mathfrak{B}, \hat{\varphi} \uparrow \mathbb{1} \otimes \mathfrak{B}, \hat{\alpha} \uparrow \mathbb{1} \otimes \mathfrak{B})$, is isomorphic to the $\left(\frac{1}{2}, \frac{1}{2}\right)$-Bernoulli system.

Proof. a) This follows in the same way as the dilation property of this system using the facts that the dilations $\left(\mathfrak{C}, \psi_{1}, \alpha_{1} ; i_{1}, R\right)$ and $\left(\mathfrak{A} \otimes \bar{\otimes}, \varphi \otimes \varphi_{2}, \alpha_{2} ; i_{2}, Q\right)$ both have the Markov property and that furthermore

$$
\hat{\mathfrak{U}}_{0,1, \ldots, n}=\mathfrak{C}_{0,1, \ldots, n} \bar{\otimes} \mathfrak{B}_{1,2, \ldots, n} .
$$


b) We want to show $\bigcap_{n \in \mathbb{N}} \hat{\alpha}^{-n}\left(\hat{\mathfrak{A}}^{>}\right)=\mathbb{C} \mathbb{1}$. Since for $n \geqq 1$ we have

$$
\hat{\alpha}^{-n}\left(a\left(f_{1}\right) \otimes \mathbb{1}\right)=e^{i n \omega} a\left(U^{-n} f_{1}\right) \otimes \prod_{l=1}^{n} \bar{\sigma}^{-l}\left(W^{*}\right),
$$

it follows that

$$
\hat{\alpha}^{-n}\left(\hat{\mathfrak{U}}^{>}\right) \subseteq \alpha_{1}^{-n}\left(\mathfrak{C}^{>}\right) \bar{\otimes} \mathfrak{B}_{(-\infty,-1]},
$$

and furthermore

$$
\bigcap_{n \in \mathbb{N}} \hat{\alpha}^{-n}\left(\hat{\mathfrak{U}}^{>}\right) \subseteq \mathbb{1} \otimes \mathfrak{B}_{(-\infty,-1]},
$$

because $\left(\mathfrak{C}, \psi_{1}, \alpha_{1} ; \mathfrak{C}^{>}\right)$is a generalized $K$-system.

The restriction of $\hat{\alpha}$ to the center $\mathbb{1} \otimes \mathfrak{B}$ induces the automorphism $\operatorname{Id} \otimes \bar{\sigma}$. Obviously $(\mathfrak{B}, v, \bar{\sigma})$ is isomorphic to the $\left(\frac{1}{2}, \frac{1}{2}\right)$-Bernoulli system.

From the theory of Bernoulli shifts [10] it is known that $\mathfrak{B}_{(-\infty,-1]}$ is an expanding subalgebra for the system $(\mathfrak{B}, v, \bar{\sigma})$. So we finally obtain

$$
\bigcap_{n \in \mathbb{N}} \hat{\alpha}^{-n}\left(\hat{\mathfrak{U}}^{>}\right) \subseteq \bigcap_{n \in \mathbb{N}} \hat{\alpha}^{-n}\left(\mathbb{1} \otimes \mathfrak{B}_{(-\infty,-1]}\right)=\mathbb{C} \cdot \mathbb{1}
$$

To show that ( $\left.\hat{\mathfrak{U}}, \hat{\varphi}, \hat{\alpha} ; \hat{\mathfrak{U}}^{>}\right)$is not weakly reversible we argue in the same way as for $\left(\mathfrak{C}, \psi_{1}, \alpha_{1} ; \mathfrak{C}^{>}\right)$. Put $q(x):=\hat{\varphi}\left(x^{*} x\right)^{1 / 2}$ for all $x \in \hat{\mathfrak{A}}$ and define $y:=a\left(f_{1}\right) \otimes \mathbb{1}=i(a)$. Then one computes for $n \geqq 1, q\left(\left[\hat{\alpha}^{-n}(y), y\right]\right)=2 \eta\left(1-e^{-\mu \cdot n}\right)^{1 / 2}$, while weak reversibility would imply $q\left(\left[\hat{\alpha}^{-n}(y), y\right]\right) \rightarrow 0$ for $n \rightarrow \infty$.

The investigation of dilations of the Bloch evolution will be carried on in a subsequent paper [9].

\section{References}

1. Bratteli, O., Robinson, D.W.: Operator algebras and quantum statistical mechanics. New York, Heidelberg, Berlin: Springer 1981

2. Emch, G.G.: Generalized $K$-flows. Commun. Math. Phys. 49, 191-215 (1976)

3. Emch, G.G.: Minimal dilations of $C P$-flows. In: Lecture Notes in Mathematics, Vol. 650, pp. 156-159. Berlin, Heidelberg, New York: Springer 1978

4. Emch, G.G., Albeverio, S., Eckmann, J.-P.: Quasi-free generalized K-flows. Rep. Math. Phys. 13, 73-85 (1978)

5. Emch, G.G., Varilly, J.C.: On the standard form of the Bloch equation. Lett. Math. Phys. 3, 113-116 (1979)

6. Evans, D.E.: Completely positive quasi-free maps on the CAR algebra. Commun. Math. Phys. 70, 53-68 (1979)

7. Kern, M., Nagel, R., Palm, G.: Dilations of positive operators: Construction and ergodic theory. Math. Z. 156, 256-277 (1977)

8. Kümmerer, B.: Markov dilations on $W^{*}$-algebras (to appear)

9. Kümmerer, B., Schröder, W.: A theory of Markov dilations for the spin- $\frac{1}{2}$-relaxation (to appear) 
10. Parry, W.: Topics in Ergodic theory. Cambridge: Cambridge University Press 1981

11. Schröder, W.: $W^{*}-K$-systems and their mixing properties (to appear)

12. Sz.-Nagy, B., Foias, C.: Harmonic analysis of operators on Hilbert space. Amsterdam: North Holland 1970

13. Varilly, J.C.: Dilation of a non-quasifree dissipative evolution. Lett. Math. Phys. 5, 113-116 (1981)

Communicated by H. Araki

Received August 30, 1982

Note added in proof. In the mean time a Markov dilation has been obtained also for the continuous form of the Bloch evolution. An account will appear in the proceedings of the "Workshop on Quantum Probability and Applications", Lecture Notes in Mathematics, Springer-Verlag. 